\title{
Genetic dissent and individual compromise
}

\section{Citation}

Haig, David. 2014. “Genetic Dissent and Individual Compromise." Biol Philos 29 (2) (January 25): 233-239. doi:10.1007/s10539-013-9418-7.

\section{Published Version}

doi:10.1007/s10539-013-9418-7

\section{Permanent link}

http://nrs.harvard.edu/urn-3:HUL.InstRepos:24981604

\section{Terms of Use}

This article was downloaded from Harvard University's DASH repository, and is made available under the terms and conditions applicable to Open Access Policy Articles, as set forth at http:// nrs.harvard.edu/urn-3:HUL.InstRepos:dash.current.terms-of-use\#OAP

\section{Share Your Story}

The Harvard community has made this article openly available.

Please share how this access benefits you. Submit a story.

Accessibility 


\title{
Genetic dissent and individual compromise
}

\author{
David Haig \\ Department of Organismic and Evolutionary Biology, \\ Harvard University, 26 Oxford Street, \\ Cambridge MA 02138.
}

\begin{abstract}
Organisms can be treated as optimizers when there is consensus among their genes about what is best to be done, but genomic consensus is often lacking, especially in interactions among kin because kin share some genes but not others. Grafen adopts a majoritarian perspective in which an individual's interests are identified with the interests of the largest coreplicon of its genome, but genomic imprinting and recombination factionalize the genome so that no faction may predominate in some interactions among kin. Once intragenomic conflicts are recognized, the individual organism can be conceptualized as an arbiter among competing interests within a collective. Organismal adaptation can be recognized without phenotypes being optimized.
\end{abstract}

Keywords: coreplicon, formal Darwinism, genomic imprinting, intragenomic conflict, recombination, strategic gene

Grafen (2013) aims to reconcile population genetics and adaptationism (the intellectual discipline that seeks to discover and explain good design in nature). At the core of his project is an optimization program in which an implicit optimizer selects the value of an instrument from a feasible set. The maximand is a function that defines the degree of success of each choice within the set. Grafen's feasible set is comprised of phenotypes of individuals and his maximand is 
individual (inclusive) fitness. In other words, Grafen chooses the individual to occupy the role of optimizer.

Many would welcome a formal justification for treating individuals rather than genes as optimizers. First, organisms are salient objects in human perception whereas genes are hidden. Second, most organismal phenotypes are products of interactions among many genes and it is difficult to ascribe particular aspects of phenotype to particular genes. Third, organisms exhibit exquisite adaptation to their environments and superb physiological and anatomical design, and these features of the natural world require explanation.

Despite the above considerations, this commentary will promote the gene as an alternative candidate to perform the role of optimizer. From this perspective, each allele's phenotypic effects are its instrument, the feasible set is defined by the set of alleles under consideration, and the maximand is a measure of genic fitness. The individual emerges as a collective entity whose behavior is influenced by multiple agents, sometimes with conflicting maximands. Individuals may be well-adapted to their environments without there being any property of the individual that is optimized by natural selection.

Grafen's individual fitness is, in fact, a measure of genic fitness, albeit a maximand that is shared by most genes of the genome, and Grafen (2006) has already incorporated intragenomic conflicts into his formal Darwinism project. Therefore, my commentary reflects no deep disagreement with Grafen's laudable goals, but rather shifts the focus from genic consensus to aspects of genic dissent.

\section{Genes as strategists}

Gardner and Welch (2011) and Haig (2012) have presented formal treatments of genes as optimizing agents. The former define the genetic agent as a gene token whereas the latter defines the agent as a set of interacting tokens. This leads to 
superficially conflicting conclusions. Consider a gene token that performs an act that confers a replicative benefit on an identical gene token. Gardner and Welch (2011) view this as selfless behavior because the token that acts does not 'personally' benefit. Haig (2012) views this as self-directed behavior because his agent was defined to encompass both the tokens that act and the replicas that benefit. This commentary will adopt the latter formalism in which the 'strategic

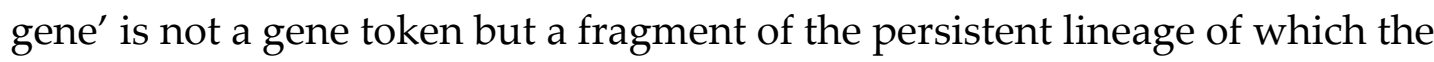
token is a temporary home. In this view, gene tokens are ephemeral vehicles of potentially immortal replicators (Dawkins 1976).

There can be no selection without choice. Selection is concerned with relative, rather than absolute, fitness because selection always involves a choice among alternatives. In natural selection, the environment chooses among alleles based on their phenotype. All things that differ between alleles comprise the phenotype subject to selection (although some differences may not influence the choice). All things that are shared by the alleles comprise the environment that selects. The environment includes other genes that are encountered with equal frequency by the two alleles, even the other allele at the same locus in a randomly mating population (Williams 1966, p. 58; Sterelny \& Kitcher 1988, p. 345). If two alleles encounter factors (be these genes, organisms, or physical factors) with different frequency, then the different frequencies are part of the phenotype subject to selection. Neither phenotype nor environment is assumed to be constant.

A bridge between population genetics and game theory can be constructed by a simple relabeling of terms. Any single-locus model in population genetics can be interpreted as a game in which alleles are the players and allelic effects are the strategies. The feasible set consists of the phenotypes of all alleles considered in the model. Multilocus models can then be viewed as strategic interactions between games played at each of the individual loci. The optimization criterion is 
success in competition with other members of the feasible set. This criterion does not always correspond to the best outcome that could be achieved in the absence of competition. Genes can be trapped in games with 'self-defeating' equilibria (e.g., Prisoners' dilemmas; Traulsen \& Reed 2012).

Genes can also be considered strategists in adaptationist models. Often such models derive an optimal phenotype by asking what phenotypic value resists invasion by all rare alternatives. In these models, different phenotypic values can be identified with the strategies of alternative alleles. The optimal phenotype corresponds to the evolutionarily stable strategy (ESS) of the phenotypic game. When the underlying phenotype is continuous, such models implicitly assume an infinite set of alleles generated by mutation; infinite because differences among alleles are infinitesimal.

Population-genetic and adaptationist models sometimes predict different equilibrium phenotypes. This is unsurprising because the models have different feasible sets and therefore need not have the same optimal instrument. Which approach is preferable for understanding a particular evolutionary problem depends on which approach provides a better model of the feasible set of interest (Haig 1999). This is often a question of implicit timescale, of whether there has been sufficient time to sample a large number of mutations. Eshel (1996) has shown that the two kinds of models have different concepts of equilibrium and address different kinds of questions, rather than one approach being superior to the other.

\section{Overdominance}

Grafen considers overdominance to be a problematic case for the individual as optimizer, because an individual of optimal phenotype inevitably produces 
offspring of suboptimal phenotype. By contrast, the gene as optimizer handles this case with ease.

Overdominance occurs when heterozygotes have higher fitness than either homozygote. Overdominance has been viewed as incompatible with gene selectionism because fitnesses are properties of diploid genotypes rather than genes (Sober \& Lewontin 1982; Lloyd 2005) or as fully compatible because genic fitnesses may be context-dependent (Maynard Smith 1987; Sterelny \& Kitcher 1988). Genic and genotypic analyses are both valid, but parse a common selective process in different ways (Waters 2005).

Consider the paradigmatic case of sickle-cell anemia from a genic perspective. In a random mating population, alleles $A$ and $S$ encounter environments $A$ and $S$ with frequencies $p$ and $(1-p)$. The genic fitness of $A$ relative to $S$ is

$$
\frac{W_{A}}{W_{S}}=\frac{p w_{A A}+(1-p) w_{A S}}{p w_{A S}+(1-p) w_{S S}}
$$

where $w_{A A}, w_{A S}$, and $w_{s s}$ are the genotypic fitnesses. The equilibrium frequency $\hat{p}$ can be found by setting $W_{A}=W$ s. At this equilibrium, there is no further evolution by natural selection because the genes do not differ in fitness, even though the population contains individuals with different genotypic fitnesses.

From a game-theoretic perspective, the feasible set consists of the strategies 'produce hemoglobin- $S$ ' or 'produce hemoglobin- $\mathrm{A}$ ' and the maximand averages across gene copies. $A$ and $S$ can be conceptualized as playing a version of the Hawk-Dove game in which both strategies are maintained at equilibrium (Maynard Smith 1987; Sterelny \& Kitcher 1988).

\section{Intragenomic conflict and genic consensus}

Cosmides \& Tooby (1981) wrote it is "useful to divide the genome into fractions whose defining rule is that fitness of all genes in the set is maximized in the same 
way. Such a set of genes that replicates together will be called a coreplicon." Their examples of coreplicons included autosomal genes, sex chromosomes, and organellar genomes. Members of different coreplicons come into conflict when they are inherited by different sets of recipients and can influence the distribution of benefits among recipients (Burt \& Trivers 2006; Grafen 2006).

Grafen $(2006,2013)$ identifies individual fitness with consensus genic fitness. His argument can be made explicit: the individual emerges as an optimizer if all, or most, of the individual's genes share the same maximand. When an organismal phenotype is determined by genes belonging to a single coreplicon, the same phenotypic value optimizes the genic fitness of each and every member of the coreplicon. When the phenotype is determined by members of different coreplicons with different optimal instruments, Grafen (2006) proposes that the interests of the largest coreplicon will usually prevail. It is the maximand of this ruling party that he interprets as individual fitness.

Membership of a single coreplicon is too stringent a requirement for the existence of genic consensus. For many aspects of phenotype, genes of different coreplicons favor the same outcomes because they share a common vehicle (the individual organism). On the other hand, the criteria of coreplication and shared maximand need not coincide when coreplicons recombine, because different elements of a coreplicon will have different distributions among kin. Relatives share some genes but not others. For this reason, social interactions among kin have the potential to disrupt genic consensus within coreplicons. A coreplicon could be redefined as a set of genes with the same maximand but the concept would lose much of its value if each local region of a chromosome possessed a unique maximand. 


\section{Genomic imprinting and conditional strategies}

Suppose that a child performs an act of benefit $B$ to itself but cost $C$ to its mother. Hamilton's rule suggests the act will be favored by natural selection if $B>r C$ where $r$ is a measure of relatedness. A gene of maternal origin in the child is nearly certain to have an identical-by-descent copy in the mother whereas a gene of paternal origin is almost certain not to have such a copy in the mother (assuming a large outbred population). If the feasible set is restricted to unimprinted strategies - strategies constrained to be the same whether a gene is maternally or paternally derived - then the appropriate value of $r$ is an average of the two probabilities, $r=1 / 2$. The optimal instrument will be a compromise between matrilineal and patrilineal interests.

Suppose, instead, that the feasible set contains imprinted strategies in which a gene's expression is conditional on its parental origin. The appropriate values of $r$ are now $r_{\mathrm{m}}=1$ (when maternally-derived) and $r_{\mathrm{p}}=0$ (when paternally-derived). From the individual perspective, the child's genome contains maternal and paternal factions that benefit from different outcomes in the child's interaction with its mother. From a genic perspective, the optimal strategy is to behave differently when maternally and paternally derived. Over the course of several generations, a gene lineage plays both roles. Similar arguments apply to a child's relatedness to its father, and to its interactions with all kin related to the child via genealogical links through the child's mother or father (Haig 1997; Grafen 2006).

If a child performs an act that benefits itself at a cost to its maternal grandmother, then $r=1 / 4$ for feasible sets that contain only unimprinted strategies (zeroth-order imprints); $r_{\mathrm{m}}=1 / 2$ and $r_{\mathrm{p}}=0$ for feasible sets that contains strategies conditional on parental origin (first-order imprints); but $r_{\mathrm{mgm}}=1, r_{\mathrm{mgf}}=r_{\mathrm{pgm}}=r_{\mathrm{pgf}}=$ 0 (mgm = maternal grandmother, etc.) for feasible sets that contain strategies conditional on grandparental origin (second-order imprints). Similar arguments 
apply to a child's relatedness to its other grandparents, and to kin related to the child via genealogical links through its grandparents. One could consider the possibility of third-order, fourth-order, and higher imprints (Haig 2000).

Most genes in mammals possess zeroth-order imprints. A few possess firstorder imprints. As yet, there is no clear evidence for higher-order imprints. Three factors may play a role. First, higher-order imprints are difficult to detect. Second, the selective forces favoring higher-order imprints are weak because these involve interactions with more distant kin for whom asymmetries of relatedness are slight and with whom interactions are less predictable. Third, the genome undergoes periodic reprogramming that erases most epigenetic marks inherited from previous generations (Hackett et al. 2013).

The purpose of this foray into parental and grandparental effects has been to show that genes on the same chromosome have different histories when chromosomes recombine and these histories may affect a gene's strategic interests. So-called 'green-beard effects' are another way in which social interactions may create genetic factions that favor different outcomes from the rest of the genome (Haig 1996; Grafen 2006). The unity of the individual frays as more and more agents dissent from the genic consensus.

\section{The evolutionary role of individuals}

In the formalism of the strategic gene, natural selection 'chooses' effects of allelic differences. Most aspects of organismal phenotype are part of the selective environment for each particular difference (Haig 2012). Nevertheless, organismal phenotypes evolve as a by-product of selection of differences at many loci. The adaptations that provide the classical evidence of design in nature are products of this process and are properties of individual organisms not individual genes. 
Are the organismal features evolved by natural selection optimal for individuals? In one sense, the answer is clearly no, as witnessed by somatic senescence. However, the formal Darwinism project takes as its maximand 'individual fitness' which entails the production of new individuals, not just somatic survival. Although reproductive success is a measure of genic fitness, Grafen posits that most genes of an individual share a single maximand that can be conceptualized as individual fitness. He does not dispute that exceptions exist to this genic consensus. The key questions become the degree of dissent and the phenotypic power of dissenting minorities. When are the effects of genes with non-conforming maximands sufficiently negligible to be ignored?

Two resolutions can be posited for the tension between viewing genes or individuals as optimizing agents. The first would be to follow Grafen's (2006) lead and argue that intragenomic conflicts can, for most purposes, be ignored because there is an effective consensus within individuals with respect to most aspects of phenotype. Of particular interest are genomic mechanisms that restrict the strategic options available to genes and thus align the interests of genes within the genome. Examples include free recombination that prevents the formation of self-serving coalitions that could conspire against the collective (Haig \& Grafen 1991) and the erasure of imprints that limits the information genes possess about their past (Hackett et al. 2013). Broad consensus is particularly plausible for phenotypes that do not directly involve interaction with kin. A kidney is an exquisite adaptation in a way that a placenta is not.

The second resolution would be to view the individual, not as an optimizer, but as an adaptive compromise among competing interests. If multiple agents, with disparate maximands, jointly determine the value of an instrument, then the instrument is unlikely to optimize any particular property of the collective. Successful organismal lineages will be those in which the 'body politic' (Haig 
2006) has evolved mechanisms, by gene-level selection, that allow effective functioning despite internal conflicts. The more effective the management of conflicts, the more successful the lineage ceteris paribus. An individual can be well-adapted, even if there is nothing particular it optimizes.

Collective entities can make decisions despite internal conflicts. Individuals are not demeaned by viewing each as a nexus of decision and responsibility that must act in the world despite multiple internal and external agents clamoring to be heard (Haig 2011). Instead of being slaves to a rigid consensus, individuals are liberated by having to choose.

Acknowledgements: The manuscript has benefited from the comments of Samir Okasha and Eneida Pardo.

Burt A, Trivers R (2006) Genes in conflict. Harvard University Press, Cambridge, MA.

Cosmides LM, Tooby J (1981) Cytoplasmic inheritance and intragenomic conflict. J Theor Biol 89:83-129.

Dawkins R (1976) The selfish gene. Oxford University Press, Oxford.

Eshel I (1996) On the changing concept of evolutionary population stability as a reflection of a changing point of view in the quantitative theory of evolution. J Math Biol 34:485-510.

Gardner A, Welch JJ (2011) A formal theory of the selfish gene. J Evol Biol 24:1801-1813.

Grafen A (2006) Optimization of inclusive fitness. J Theor Biol 238:541-563. Grafen A (2013) The formal darwinism project in outline. Biol Philos, in press. 
Hackett JA, Sengupta R, Zylicz JJ, Murakami K, Lee C, et al (2013) Germline DNA demethylation dynamics and imprint erasure through 5hydroxymethylcytosine. Science 339:448-452.

Haig D (1996) Gestational drive and the green-bearded placenta. Proc Natl Acad Sci 93:6547-6551.

Haig D (1997) Parental antagonism, relatedness asymmetries, and genomic imprinting. Proc R Soc B 264:1657-1662.

Haig D (1999) Multiple paternity and genomic imprinting. Genetics 151:12291231.

Haig D (2000) Genomic imprinting, sex-biased dispersal, and social behavior. Ann NY Acad Sci 907:149-163.

Haig D (2006) Intragenomic politics. Cytogenet Genome Res 113:68-74.

Haig D (2011) Sympathy with Adam Smith and reflexions on self. J Econ Organ Behav 77:4-13.

Haig D (2012) The strategic gene. Biol Philos 27: 61-479.

Haig D, Grafen A (1991) Genetic scrambling as a defence against meiotic drive. J Theor Biol 153:531-558.

Lloyd E (2005) Why the gene will not return. Philos Sci 72:287-310. Maynard Smith J (1987) How to model evolution. In: Dupre J, ed. The latest on the best: essays on evolution and optimality. MIT Press, Cambridge, MA, pp. 119-131.

Sober E, Lewontin RC (1982) Artifact, cause, and genic selection. Philos Sci 49:157-180.

Sterelny K, Kitcher P (1988) The return of the gene. J Philos 85:339-361.

Traulsen A, Reed FA (2012) From genes to games: cooperation and cyclic dominance in meiotic drive. J Theor Biol 299:120-125. 
Waters CK (2005) Why genic and multi-level selection theories are here to stay. Philos Sci 72:311-333.

Williams GC (1966) Adaptation and natural selection. Princeton University Press, Princeton. 